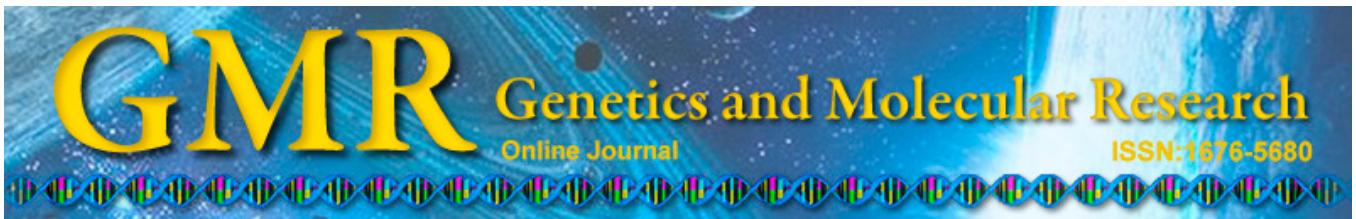

\title{
Genetic structure and diversity in natural and stocked populations of the mandarin fish (Siniperca chuatsi) in China
}

\author{
M. Yang*, C. Tian*, X.-F. Liang, H. Zheng, C. Zhao and K. Zhu \\ College of Fisheries, Key Laboratory of Freshwater Animal Breeding, \\ Ministry of Agriculture, Huazhong Agricultural University, \\ Freshwater Aquaculture Collaborative Innovation Center of Hubei Province, \\ Wuhan, Hubei, China \\ *These authors contributed equally to this study. \\ Corresponding author: X.-F. Liang \\ E-mail: xufang_liang@hotmail.com
}

Genet. Mol. Res. 14 (2): 5153-5160 (2015)

Received August 28, 2014

Accepted January 14, 2015

Published May 18, 2015

DOI http://dx.doi.org/10.4238/2015.May.18.5

\begin{abstract}
The Chinese perch, or mandarin fish (Siniperca chuatsi), is a freshwater fish that is endemic to East Asia. In this study, we investigated the genetic diversity and structure of nine natural mandarin fish populations (from the Yangtze River and Amur River basins) and six hatchery stocks (from central and south China) using microsatellite markers. The results show that the genetic diversity of the Yangtze River populations was high and stable, and genetic differences between them were not significant. In contrast, a low level of genetic diversity and strong genetic structure were detected in the Amur River population. These results suggest that the Yangtze River region and the Amur River region should be treated as two separate units in conservation programs. The hatchery stocks exhibited low genetic diversity and significant genetic differentiation compared to natural populations; this may result in a significant impact on the species if escape events occur. Therefore,
\end{abstract}


a scientific aquaculture management strategy is necessary for the longterm development of hatcheries.

Key words: Genetic diversity; Genetic structure; Mandarin fish; Microsatellite markers

\section{INTRODUCTION}

The Chinese perch or mandarin fish (Siniperca chuatsi) is a freshwater fish species endemic to East Asia. It is found in most river systems of eastern China, particularly in large, slow-moving rivers (Liang, 1996). However, its natural resources have reduced dramatically since the 1970s, due to habitat degradation and anthropogenic effects (e.g., damming, water pollution, and overfishing) (Li, 1991; Zhang and Zhao, 1999). In the wild, mandarin fish are all but extinct in some regions of north China (Yang, 2003).

In addition, the development of the aquaculture industry and the absence of a scientific or rational management of hatchery stocks is also a threat to the species (Wang et al., 2006). Hence, it is becoming increasingly important to understand the genetic diversity and structure of natural and hatchery populations (Liang, 1996). However, the number of samples and populations used in previous studies has been insufficient for robust quantitative assessments; therefore, information regarding the detailed genetic structure of the mandarin fish is still limited.

In the present study, we selected nine natural populations (from five tributaries and one lake of the Yangtze River basin) and six artificial populations (from the main hatcheries of central and south China) to analyze their genetic diversities and structures using microsatellite markers. This information may provide a scientific basis for the conservation and sustainable use of natural populations, and the high-efficiency utilization of the species' germplasm resources in breeding programs.

\section{MATERIAL AND METHODS}

A total of 444 specimens were analyzed, including 273 natural samples from the Yangtze River basin (Yuan River, Xiang River, Ganjiang River, Lushui River, and Dongting Lake) and the Amur River basin (Songhua River). We collected six natural populations from different sites of the Yuan River, the Lushui River, and Dongting Lake (Yuan River, YUAN-A and YUAN-B; Lushui River, LU-A and LU-B; Dongting Lake, DON-A and DON-B). A total of 171 stock specimens were collected from different hatcheries in central and southern China (Central: Hubei Province, Hunan Province, and Jiangxi Province; South: Guangdong Province). As Guangdong Province is the main region for farming mandarin fish in China, we collected three stock populations from different hatcheries in this region (GD-A, GD-B, and GD-C) (Figure 1A). Total genomic DNA was extracted from fin clips using a TIANamp Genomic DNA Kit (Tiangen, Beijing China). DNA was adjusted to a volume of $100 \mathrm{ng} / \mu \mathrm{L}$ and stored at $-20^{\circ} \mathrm{C}$.

Twelve microsatellite markers were used to estimate the genetic diversity and structure of these populations. Nine primers (SO374, SS55, SS62, SC01, SC80, Sin138, Sin142, Sin166, and SK524) were taken from Qu et al. (2012) and Huang et al. (2013), and the other three primers (FC076, PY55, and SK608) were developed from genomic sequences of $S$. chuatsi in our laboratory: FC076 (F, 5'-TACCCCAGTCGTGTCCCTT-3'; R, 5'-CTTTCCTTATTTATTGACTC-3'); PY55 (F, 5'-TGGGTAGGCTTCATGTGGTA-3'; R, 
5'-TGCCACCTTTAGATTTCAGC-3'); and SK608 (F, 5'-GTGGTTTCTACATTTGGGTC-3'; R, 5'-CAGTCAACATTACAGAACCTCA-3'). Microsatellites were multiplexed with up to three fluorescently labeled primers. The polymerase chain reaction (PCR) system and thermal cycling conditions were conducted according to Huang et al. (2013). PCR products were separated using an automatic capillary sequencer (ABI 3130 Genetic Analyzer, Applied Biosystems) at Sangon Biotech (Shanghai, China). Fragment sizes were determined using the GeneMapper $^{\circledR}$ software version 4.0 (Applied Biosystems), by comparing with a GeneScan ${ }^{\mathrm{TM}}$ 500 ROX $^{\mathrm{TM}}$ (Applied Biosystems) internal size standard.

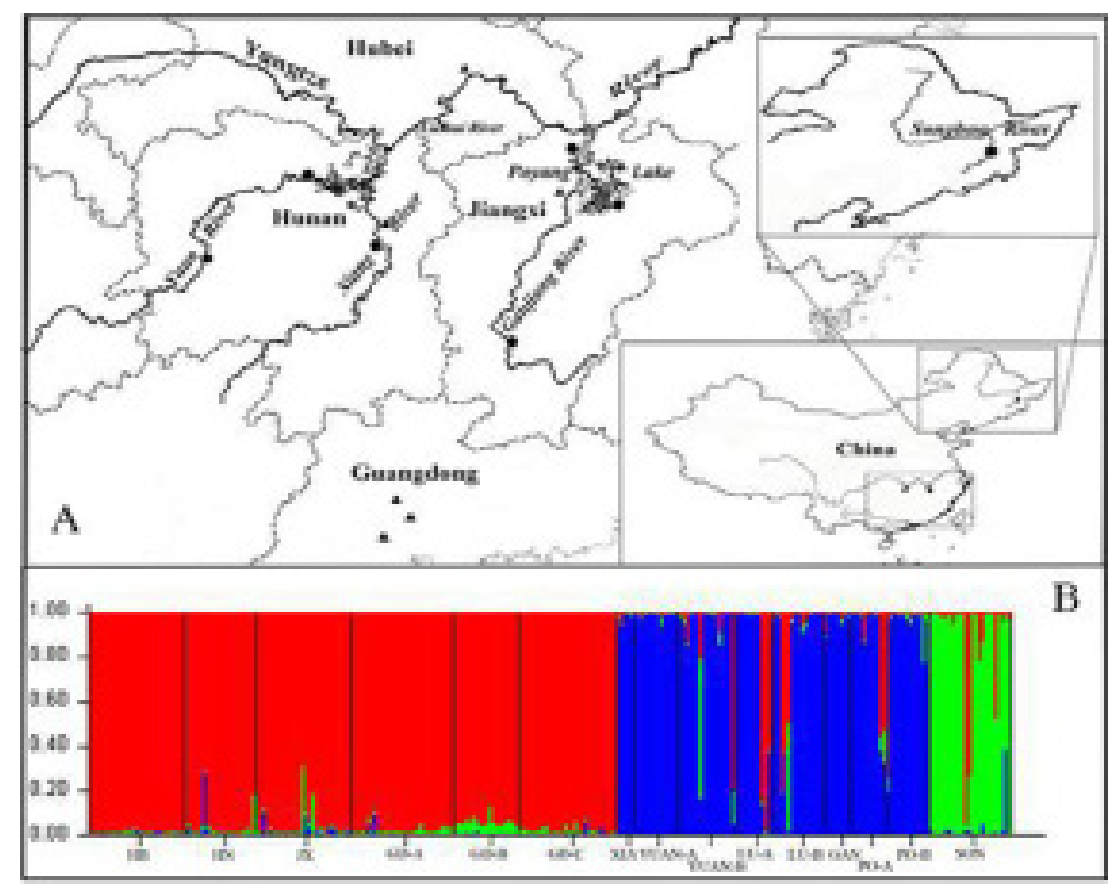

Figure 1. A. Map showing sampling locations. Circles denote wild samples and triangles indicate hatchery samples. B. Population genetic structure analysis with the Structure 2.2 software. Each individaul is represented by a single vertical line divided into three colours. Each color represents one gene pool, and the length of the coloured segment shows the individual's estimated proportion of membership in that gene pool. Black lines separate populations that are labelled below the figure.

The presence of null alleles, large allele drop-outs, and scoring errors was evaluated using MicroChecker version 2.2.3 (Van Oosterhout et al., 2004). Genepop version 4.0 (Raymond and Rousset, 1995) was used to test the Hardy-Weinberg equilibrium (HWE) and linkage disequilibrium. POPGENE version 1.3 (Yeh, 1997) was used to estimate the observed $\left(H_{\mathrm{O}}\right)$ and expected $\left(H_{\mathrm{E}}\right)$ heterozygosities. An analysis of molecular variance (AMOVA) was conducted using Arlequin 3.11 (Excoffier and Lischer, 2010); for this analysis, the natural populations were classified as one group and the stock populations were classified as another group. Pairwise $F_{\mathrm{ST}}$ values were also calculated by Arlequin. The population genetic structure was analyzed by Structure, version 2.2 (Pritchard et al., 2000). The most probable number of clusters in the dataset was identified based on the probability $K=2$ to 15 , with $1,000,000$ 
Markov chain Monte Carlo repetitions each and a burn-in of 10,000 iterations. Each value of $K$ was tested at least 20 times to estimate the deviation between repeated runs. The most probable $K$ value was determined according to Evanno et al. (2005).

\section{RESULTS}

Three of the twelve microsatellite markers (SC608, Sin142, and PY55) were polymorphisms and were excluded from further analyses. Of the natural populations, the Poyang Lake population exhibited the highest mean levels of genetic diversity [PO-A: $N_{\mathrm{A}}$ (average number of alleles per locus), $6.44 ; H_{\mathrm{O}}, 0.788 ; H_{\mathrm{E}}, 0.726 ;$ PO-B: $\left.N_{\mathrm{A}}, 6.33 ; H_{\mathrm{O}}, 0.752 ; H_{\mathrm{E}}, 0.716\right]$, and the Amur River population exhibited the lowest mean levels of genetic diversity (SON: $N_{\mathrm{A}}, 3.89$; $\left.H_{\mathrm{O}}, 0.471 ; H_{\mathrm{E}}, 0.451\right)$. All of the hatchery stocks exhibited low mean levels of genetic diversity $\left(N_{\mathrm{A}}, 3.15 ; H_{\mathrm{O}}, 0.424 ; H_{\mathrm{E}}, 0.398\right)$ (Table 1$)$. The $N_{\mathrm{A}}, H_{\mathrm{O}}$, and $H_{\mathrm{E}}$ values of the hatchery groups were significantly lower than those of the natural groups $(\mathrm{P}<0.05)$.

\begin{tabular}{|c|c|c|c|c|c|c|c|c|}
\hline River & Pop code & Region & Population type & $\mathrm{N}$ & $N_{\mathrm{A}}$ & $N_{\mathrm{E}}$ & $H_{\mathrm{O}}$ & $H_{\mathrm{E}}$ \\
\hline Xiang River & XIA & Yangtze River & Natural & 30 & 4.22 & 3.00 & 0.667 & 0.662 \\
\hline Yuan River & YUA-A & Yangtze River & Natural & 33 & 5.78 & 3.36 & 0.590 & 0.664 \\
\hline Yuan River & YUA-B & Yangtze River & Natural & 28 & 7.44 & 3.90 & 0.722 & 0.730 \\
\hline Lushui River & LU-A & Yangtze River & Natural & 31 & 5.78 & 4.17 & 0.606 & 0.724 \\
\hline Lushui River & LU-B & Yangtze River & Natural & 36 & 6.33 & 3.78 & 0.729 & 0.734 \\
\hline Ganjiang River & GAN & Yangtze River & Natural & 28 & 5.22 & 3.14 & 0.694 & 0.686 \\
\hline Poyang Lake & PO-A & Yangtze River & Natural & 31 & 6.44 & 3.77 & 0.788 & 0.726 \\
\hline Poyang Lake & PO-B & Yangtze River & Natural & 31 & 6.33 & 3.74 & 0.752 & 0.716 \\
\hline Songhua River & SON & Amur River & Natural & 25 & 3.89 & 2.09 & 0.471 & 0.451 \\
\hline Hubei Province & $\mathrm{HB}$ & Central China & Stocked & 29 & 3.00 & 1.84 & 0.410 & 0.389 \\
\hline Hunan Province & $\mathrm{HN}$ & Central China & Stocked & 22 & 3.00 & 1.81 & 0.419 & 0.375 \\
\hline Jiangxi Province & $\mathrm{JX}$ & Central China & Stocked & 29 & 4.22 & 1.88 & 0.521 & 0.418 \\
\hline Guangdong Province & GD-A & South China & Stocked & 31 & 3.22 & 1.80 & 0.369 & 0.361 \\
\hline Guangdong Province & GD-B & South China & Stocked & 30 & 2.78 & 1.98 & 0.494 & 0.372 \\
\hline Guangdong Province & GD-C & South China & Stocked & 30 & 2.67 & 1.70 & 0.356 & 0.311 \\
\hline Mean of natural group & & & & 273 & 5.72 & 4.44 & 0.651 & 0.749 \\
\hline Mean of stocked group & & & & 171 & 3.15 & 1.91 & 0.424 & 0.398 \\
\hline Total & & & & 444 & 4.69 & 2.94 & 0.521 & 0.629 \\
\hline
\end{tabular}

$\mathrm{N}=$ number of samples; $N_{\mathrm{A}}=$ average number of alleles per locus; $N_{\mathrm{E}}=$ effective number of alleles per locus; $H_{\mathrm{O}}=$ observed heterozygosity; $H_{\mathrm{E}}=$ expected heterozygosity.

The pairwise $F_{\mathrm{ST}}$ values varied from -0.014 to 0.501 , and all of the hatchery stocks exhibited significant genetic differences between each other. Samples from the Amur River also exhibited significant genetic differences from the other populations. However, no significant genetic differentiation was found between the Yangtze River populations. In the natural populations, the lowest value of Nei's genetic distance (0.079) was found between the Ganjiang River (GAN) and Poyang Lake (PO-B) populations, and the highest value (1.1841) was found between the Amur River (SON) and the Yuan River (YUA-A) populations (Table 2). The AMOVA revealed a significant difference between the natural and artificial groups ( $\mathrm{FCT}=$ $0.219, \mathrm{P}<0.01) ; 68.92 \%$ of the genetic variance was between individuals within populations, and $21.86 \%$ was between the natural and hatchery groups. 


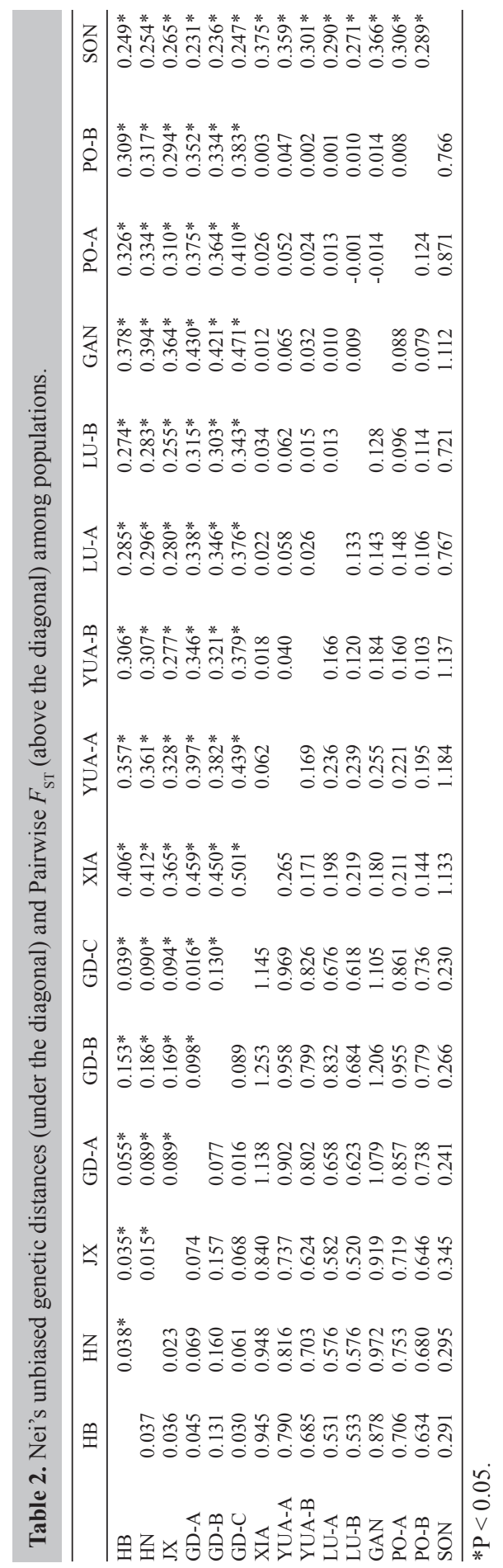


Bayesian clustering analysis revealed that the highest $\Delta \mathrm{K}$ value (83.97) was for $\mathrm{K}=$ 3 , suggesting that all of the individuals fell into three main genetic groups. All of the hatchery stocks were classified into one group, the Amur River population was classified as another group, and the Yangtze River population was the third group. Some stock individuals (sometimes consisting of $80 \%$ of the population) were found in natural populations (Figure 1B).

\section{DISCUSSION}

The levels of genetic variability in the Yangtze River populations that we found were similar to those estimated by Mei (2010) and Wu et al. (2010), suggesting that the populations of these regions are relatively stable. Genetic diversity in the Amur River samples was very low, suggesting a serious decline of the species, possibly caused by recent habitat destruction and environmental degradation. The low genetic diversity we found in the hatchery populations (both in the central and southern areas) could be explained by several factors: 1) a relatively small effective population size; 2) the effects of genetic drift during the initial stages of transplanting; and 3) intensive directional selection in breeding programs (Doebley et al., 2006; Gross and Olsen, 2010; Karlsson et al., 2010). Therefore, for the genetic improvement of hatchery stocks it is necessary to construct a broad genetic base with a large effective population size, or to regularly introduce individuals from natural populations.

All of the hatchery stocks exhibited high genetic distances and significant genetic differentiation compared to the other populations. The genetic differences between hatchery stocks may have been due to random genetic drift under the stress of artificial selection, which has been well documented in other species (Hedgecock and Sly, 1990; Desvignes et al., 2001; Li et al., 2007; Dawnay et al., 2011), and differences in their environments and management patterns would also cause them to gradually diverge (Liu et al., 2012). The difference between the natural and the artificial populations could be due to a lack of gene flow; their geographical isolation from each other was probably the cause of this. In addition, the different environmental conditions in natural and stock populations also probably contributed to their genetic differences (Liu et al., 2012).

A weak genetic structure was observed in the Xiang River, Yuan River, Lushui River, Ganjiang River, and Poyang Lake populations. All of these tributaries, and the lake, are connected to the Yangtze River, so there is little geographical isolation; the short distances between the populations increased gene flow and probably contributed to their weak genetic structure. By contrast, a strong genetic structure was found in the Yangtze River and Amur River populations; this could be explained by the geographical features of these areas. The Yangtze River and Amur River are south and north, respectively, of the Qinling Mountains and the Huaihe River, which are important geographical boundaries in China. Differences in topography and climate between the south and north may have led to these endemic genetic characteristics. Based on this evidence, we suggest that the Yangtze and Amur River populations should be managed as separate conservation units.

Fish that had been reared in hatcheries were in wild populations, according to Bayesian analysis, suggesting that some individuals may have escaped into the wild gene pool. This is important, because the escape of hatchery stocks may pose a threat to the genetic diversity and/or genetic structure of natural populations (Clifford et al., 1998; Koljonen et al., 2002; Ma et al., 2011). Therefore, to prevent escape events, it is necessary to improve the genetic management and conservation plan for this species. 
The data obtained in the present study should be regarded as preliminary, as there are many natural and hatchery resources at other locations yet to be investigated. Therefore, in order to provide a scientific management strategy for the mandarin fish, full-scale analyses should be conducted in future studies.

\section{ACKNOWLEDGMENTS}

Research supported by the Key Projects in the National Science and Technology Pillar Program During the Twelfth Five-Year Plan Period (\#2012BAD25B00), the National Natural Science Foundation of China (\#31272641, \#31172420), the National Basic Research Program of China (\#2009CB118702), the Fundamental Research Funds for the Central Universities (\#2011PY030), and the Huazhong Agricultural University Scientific and Technological Selfinnovation Foundation (\#2012SC24).

\section{REFERENCES}

Clifford S, McGinnity P and Ferguson A (1998). Genetic changes in an Atlantic salmon population resulting from escaped juvenile farm salmon. J. Fish. Biol. 52: 118-127.

Dawnay N, Dawnay L, Hughes RN, Cove R, et al. (2011). Substantial genetic structure among stocked and native populations of the European grayling (Thymallus thymallus, Salmonidae) in the United Kingdom. Conserv. Genet. 12: $731-744$

Desvignes JF, Laroche J, Durand JD and Bouvet Y (2001). Genetic variability in reared stocks of common carp (Cyprinus carpio L.) based on allozymes and microsatellites. Aquaculture 194: 291-301.

Doebley JF, Gaut BS and Smith BD (2006). The molecular genetics of crop domestication. Cell 127: 1309-1321.

Evanno G, Regnaut S and Goudet J (2005). Detecting the number of clusters of individuals using the software STRUCTURE: a simulation study. Mol. Ecol. 14: 2611-2620.

Excoffier L and Lischer HE (2010). Arlequin suite ver 3.5: a new series of programs to perform population genetics analyses under Linux and Windows. Mol. Ecol. Resour. 10: 564-567.

Gross BL and Olsen KM (2010). Genetic perspectives on crop domestication. Trends Plant Sci. 15: 529-537.

Hedgecock D and Sly F (1990). Genetic drift and effective population sizes of hatchery-propagated stocks of the Pacific oyster, Crassostrea gigas. Aquaculture 88: 21-38.

Huang W, Liang X-F, Qu C-M, Zhao C, et al. (2013). Isolation and characterization of 31 polymorphic microsatellite markers in Siniperca obscura Nichols. Conserv. Genet. Resour. 5: 153-156.

Karlsson S, Moen T and Hindar K (2010). Contrasting patterns of gene diversity between microsatellites and mitochondrial SNPs in farm and wild Atlantic salmon. Conserv. Genet. 11: 571-582.

Koljonen M-L, Tähtinen J, Säisä M and Koskiniemi J (2002). Maintenance of genetic diversity of Atlantic salmon (Salmo salar) by captive breeding programmes and the geographic distribution of microsatellite variation. Aquaculture 212: 69-92.

Li Q, Xu K and Yu R (2007). Genetic variation in Chinese hatchery populations of the Japanese scallop (Patinopecten yessoensis) inferred from microsatellite data. Aquaculture 269: 211-219.

Li S (1991). Study on the geographical distribution of Sinipercinae fishes. Chin. J. Zool. 26: 40-43.

Liang XF (1996). Study on Mandarin fish and its culture home and abroad. Sci. Technol. Inf. 23: 13-17.

Liu F, Yao J, Wang X, Repnikova A, et al. (2012). Genetic diversity and structure within and between wild and cultivated Saccharina japonica (Laminariales, Phaeophyta) revealed by SSR markers. Aquaculture 358-359: 139-145.

Ma Y, Wang C, Wang J, Yang X, et al. (2011). Genetic differentiation of wild and hatchery Oujiang color common carp: potential application to the identification of escapees. Fish. Sci. 77: 591-597.

Mei Q (2010). Analysis of genetic diversity between protospecies and cultured populations of Siniperca chuatsi based on TRAP and SSR markers. Hunan Agricultural University, Wuhan, Hubei, China.

Pritchard JK, Stephens M and Donnelly P (2000). Inference of population structure using multilocus genotype data. Genetics 155: 945-959.

Qu C, Liang XF, Huang W and Cao L (2012). Isolation and characterization of 46 novel polymorphic EST-simple sequence repeats (SSR) markers in two sinipercine fishes (Siniperca) and cross-species amplification. Int. J. Mol. Sci. 13: 9534-9544. 
Raymond $M$ and Rousset $\mathrm{F}$ (1995). GENEPOP (version 1.2): population genetics software for exact tests and ecumenicism. J. Hered. 86: 248-249.

Van Oosterhout C, Hutchinson WF, Wills DPM and Shipley P (2004). Micro-Checker: software for identifying and correcting genotyping errors in microsatellite data. Mol. Ecol. Notes 4: 535-538.

Wang W, Zhao J and Li S (2006). Genetic variation of the mitochondrial DNA control region among 5 populations of Siniperca scherzeri Steindachner in China. J. Shanghai Fish. Univ. 4: 398-402.

Wu X, Yan M and Li Z (2010). Genetic structure of mandarin fish Siniperca chuatsi in different geographic populations along the middle and lower reaches of Yangtze River Basin. Acta Hydr. Sin. 34: 843-850.

Yang S (2003). The resource utilization and diversity of the Sinipercinae fishes. Fish. Sci. Technol. Inf. 3: 121-125.

Yeh F (1997). Population genetic analysis of codominant and dominant markers and quantitative traits. Belg. J. Bot. 129: 157.

Zhang C and Zhao Y (1999). Study on the resource of mandarin fish and its rational utilization in China. Bull. Biol. 34: 9-11. 